Supplement of Weather Clim. Dynam., 1, 93-109, 2020

https://doi.org/10.5194/wcd-1-93-2020-supplement

Weather and

(C) Author(s) 2020. This work is distributed under the Creative Commons Attribution 4.0 License.

(c) (1)

L

Supplement of

\title{
The role of wave-wave interactions in sudden stratospheric warming formation
}

Erik A. Lindgren and Aditi Sheshadri

Correspondence to: Erik A. Lindgren (ealindgr@stanford.edu)

The copyright of individual parts of the supplement might differ from the CC BY 4.0 License. 


\section{Introduction}

The supporting information contains figures showing the zonal mean zonal winds for the four model runs with heating wave 1 forcing, and time evolution of the polar vortex strength during 2000 days of the model runs. The method for assessing statistical significance is also described in detail. Movies showing the absolute vorticity at $10 \mathrm{hPa}$ and $80 \mathrm{hPa}$ around the sudden stratospheric warmings (SSWs) seen in Fig. 8 are uploaded separately, and can be found at https://osf.io/3vrmel.
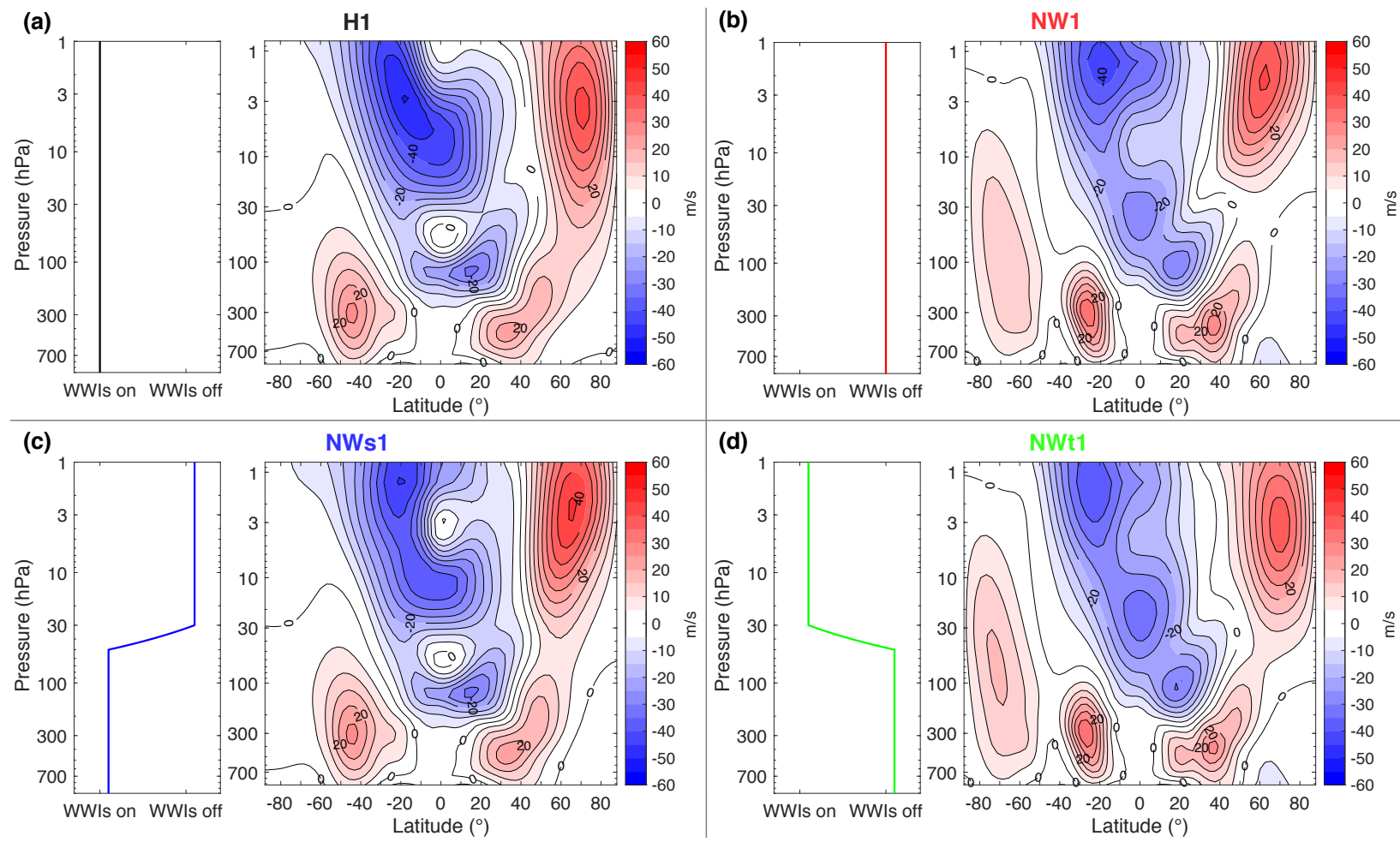

Figure S1. Zonal mean zonal winds for H1 (a), NW1 (b), NWs1 (c) and NWt1 (d). The contour interval is $5 \mathrm{~m} \mathrm{~s}^{-1}$. 

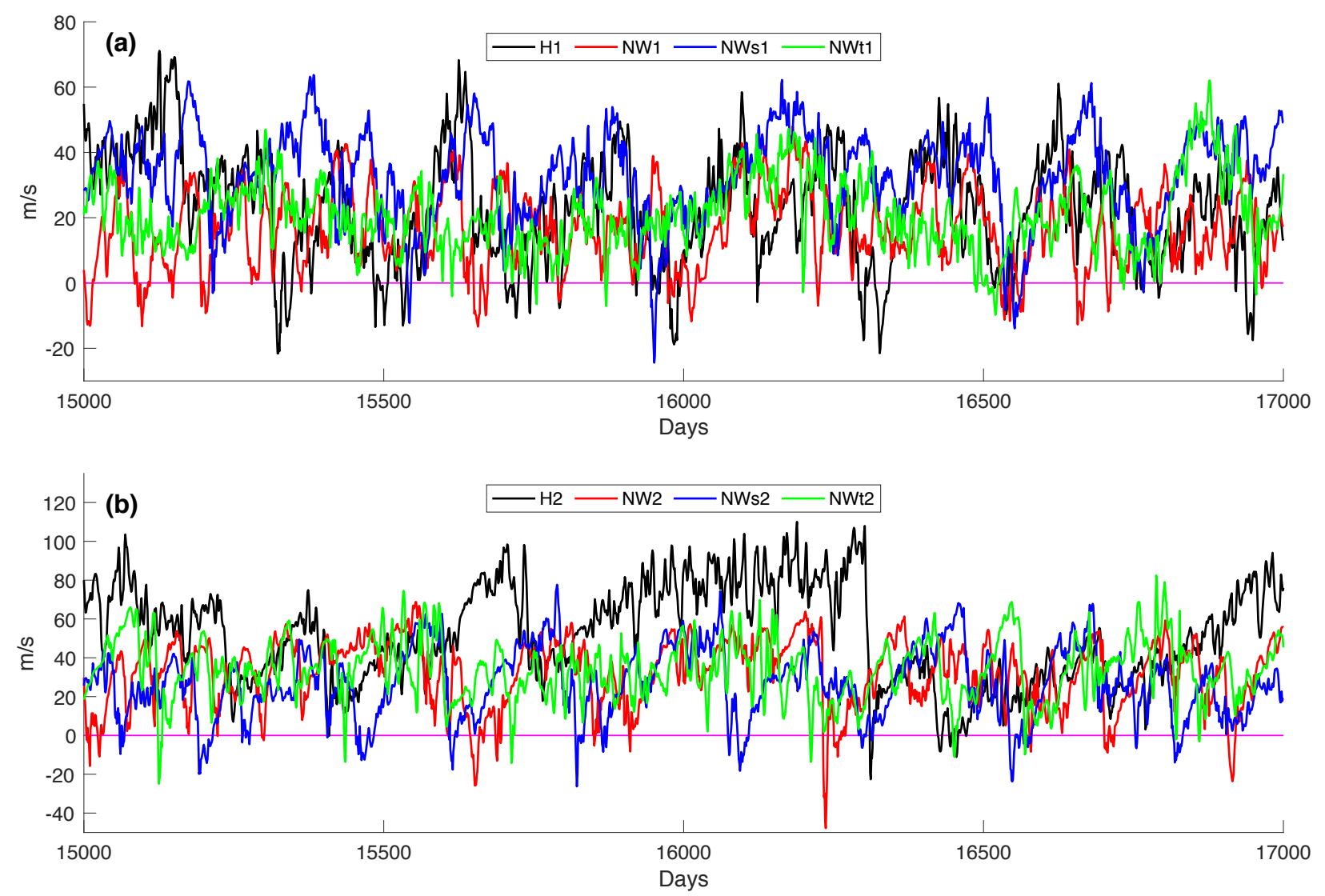

Figure S2. Zonal mean zonal wind at $10 \mathrm{hPa}$ and $60^{\circ} \mathrm{N}$ for $\mathrm{H} 1 / \mathrm{H} 2$ (black), NW1/NW2 (red), NWs1/NWs2 (blue) and NWt1/NWt2 (green) for model runs with wave 1 (a) and wave 2 (b) heating. The magenta line marks $0 \mathrm{~m} \mathrm{~s}^{-1}$.

\section{Method for assessing statistical significance}

We use a Monte Carlo approach to estimate the statistical significance of differences in SSW frequencies as well as split and displacement ratios between the model runs. The method takes observed SSW frequencies and split and displacement ratios, and finds the percentiles of frequencies and ratios that one would expect from a random process with probabilities equal to those of the observed values.

For each model run, we produce 10,000 sets of length 30,000 (equal to the number of days in the model runs). Each of the 10,000 sets is then assigned a number of "SSWs": this number is created using a random number generator where the probability of producing a SSW is equal to the SSW frequency in the model run. From the 10,000 sets we obtain the 2.5 and 97.5 percentiles of SSW frequencies corresponding to each observed SSW frequency.

We use a similar approach for splits and displacements: for each model run 10,000 sets of length equal to the number of SSWs in the model is produced. A number of "splits" is assigned to each of the 10,000 sets using the same random number generator, where the probability of producing a split is equal to the fraction of splits in 
the corresponding model run. From this we obtain the 2.5 and 97.5 percentiles of the fraction (number) of splits corresponding to each observed split fraction.

The results are shown in Table S1. If the observed SSW frequency or split fraction of a given model run is outside the 2.5 and 97.5 percentiles for a different model run the differences between these two model runs are considered statistically significant at a $95 \%$ confidence level.

\begin{tabular}{|c|c|c|c|c|c|c|c|c|}
\hline & H1 & NW1 & NWt1 & NWs1 & H2 & NW2 & NWt2 & NWs2 \\
\hline $\begin{array}{c}\text { ssWs per } \\
\text { 100 days }\end{array}$ & 0.66 & 0.82 & 0.44 & 0.31 & 0.48 & 0.51 & 0.45 & 0.66 \\
\hline $\begin{array}{c}\text { Percent } \\
\text { splits }\end{array}$ & 59 & 57 & 80 & 80 & 74 & 100 & 100 & 90 \\
\hline $\begin{array}{c}\mathbf{2 . 5 / 9 7 . 5} \\
\text { percentiles, } \\
\text { ssW freq. }\end{array}$ & 0.58 & 0.72 & 0.37 & 0.25 & 0.40 & 0.43 & 0.37 & 0.57 \\
\hline $\begin{array}{c}\text { 2.5/97.5 } \\
\text { percentiles, } \\
\% \text { splits }\end{array}$ & 53 & 52 & 73 & $\begin{array}{c}71 \\
86\end{array}$ & $\begin{array}{c}67 \\
86\end{array}$ & $\begin{array}{c}100 \\
100\end{array}$ & $\begin{array}{c}100 \\
100\end{array}$ & $\begin{array}{c}85 \\
94\end{array}$ \\
\hline
\end{tabular}

Table S1. 2.5 and 97.5 percentiles of expected SSW frequencies and percentages of splits for each model run. 